\title{
Molecular Dynamics of 18-Crown-6 Complexes with Alkali-Metal Cations and Urea: Prediction of Their Conformations and Comparison with Data from the Cambridge Structural Database
}

\author{
F.T.H. Leuwerink, ${ }^{*}$ S. Harkema, W.J. Briels, and D. Feil \\ Chemical Physics Laboratory, University of Twente, P.O. Box 217, 7500 AE Enschede, The Netherlands
}

Received 12 October 1992; accepted 27 February 1993

\begin{abstract}
Complexes of 18-crown-6 with alkali-metal cations $\left(\mathrm{Na}^{+}, \mathrm{K}^{+}\right.$, and $\left.\mathrm{Rb}^{+}\right)$, urea, and the uncomplexed crown ether were studied in vacuo with the molecular dynamics method. Conformational data from these calculations (simulation times in the range from 6-15 ns) was compared with information from the Cambridge Structural Database. Despite the differences in condition between the simulations and the solid state, a number of interesting similarities are observed. (c) 1993 by John Wiley \& Sons, inc.
\end{abstract}

\section{INTRODUCTION}

Inclusion compounds are the subject of much chemical research. In particular, the macrocyclic polyethers (crown ethers), discovered by C.J. Pedersen ${ }^{1}$ in 1967, are used for selective bonding of various cations and neutral molecules.

Because molecular mechanical and dynamical simulation techniques have been shown to be a powerful tool for probing the properties of flexible large molecules, a vast number of statistical mechanical calculations have been performed on a variety of supramolecular complexes. For many of these studies, the prototypical crown ether 1,4,7,10,13,16-hexaoxacyclooctadecane (18-crown-6) was used. For example, the experimentally observed preference of 18-crown-6 for the potassium cation, above sodium and rubidium, in aqueous solution was reproduced by means of free energy calculations. ${ }^{2}$ Straatsma et al. ${ }^{3}$ used 18-crown- 6 to demonstrate the applicability of the perturbation method and the thermodynamic integration technique to evaluate free energy differences for systems with multiple rotational isomeric states. Recently, Sun and Kollman ${ }^{4}$ used 18-crown-6 as a test case for conformational sampling and ensemble generation by molecular dynamics simulations.

In the study reported here, different complexes of 18-crown- 6 are simulated. The flexible 18-crown- 6 molecule is known to adopt different conformations when complexing different guest species. The purpose of this study is to predict for a number of complexes, on the basis of in vacuo molecular dynamic

\footnotetext{
*Author to whom all correspondence should be addressed.
}

simulations, what conformation is assumed in the solid state. For this, the results of the simulations are compared with information from the Cambridge Structural Database (CSD) ${ }^{5}$ The search in the Database was confined to unsubstituted 18-crown-6 molecules without disorder in the crystal structures.

\section{METHOD}

All simulations were carried out by usng the molecular mechanical simulation package GROMOS. ${ }^{6}$ Its potential-energy function contains harmonic potentials for bond stretching, bond-angle bending and improper dihedral bending interactions, a series expansion for the torsional energy, and Lennard-Jones and Coulomb potentials for the nonbonded interactions:

$$
\begin{aligned}
E_{\mathrm{pot}}=\sum \frac{k_{s}}{2}\left(l-l_{0}\right)^{2} & +\sum \frac{k_{b}}{2}\left(\theta-\theta_{0}\right)^{2} \\
+\sum \frac{k_{i}}{2}\left(\xi-\xi_{0}\right)^{2} & +\sum \frac{V_{n}}{2}\{1+\cos (n \omega-\delta)\} \\
+ & \sum \frac{C_{12}}{r^{12}}-\sum \frac{C_{6}}{r^{6}}+\sum \frac{q_{i} q_{j}}{4 \pi \varepsilon_{0} r}
\end{aligned}
$$

The parameters for the 18-crown-6 molecule were taken from the AMBER all-atom force field ${ }^{7}$ with a $50 \%$ scaling for the nonbonded 1,4 interactions. Atomic charges for the crown ether and the Lennard-Jones parameters for the metal-ions were taken from a previous study. ${ }^{8}$ For urea, the potential of Hagler et al. ${ }^{9}$ was used, supplemented with force constants from the GROMOS force field. ${ }^{6}$ This was in accordance with a previous study on the interface between water and crystalline urea. ${ }^{10}$ 
The SHAKE procedure ${ }^{11}$ was applied to constrain all the hydrogen-carbon and hydrogen-nitrogen bond lengths. The simulations were done in the gas phase in the NVE ensemble at $298 \mathrm{~K}$ and used a time step of $0.002 \mathrm{ps}$. The fluctuations in the temperature amounted to approximately $30 \mathrm{~K}$. The coordinates were saved every $0.5 \mathrm{ps}$.

To be able to identify the different conformations of the crown ether molecule, the following convention is used. The dihedral angles in the crown ether molecule are labeled + for the gauche $(+)$ position, i.e., angles between 0 and $2 \pi / 3,-$ for the gauche $(-)$ position, i.e., angles between 0 and $-2 \pi / 3$, and 0 for the remaining trans positions. The labels are grouped into triplets, in which the second symbol represents the torsion angle around the central bond of the $\mathrm{O}$ $\mathrm{CH}_{2}-\mathrm{CH}_{2}-\mathrm{O}$ unit. In what follows, the term conformation refers to a particular combination of dihedral angles using this notation.

\section{RESULTS}

\section{8-Crown-6: $\mathrm{K}^{+}$Complex}

The first simulation that was performed was that of the 18-crown-6-potassium complex starting from the $\mathrm{D}_{33 d}$ conformation (code: $0+00-00+00-00+0$ $0-0$ ). During this simulation, which had a total length of $8 \mathrm{~ns}$, no dihedral transitions were observed. The entire simulation the crown ether stayed in the $\mathrm{D}_{3 d}$ conformation. To test the preference for the $\mathrm{D}_{3 d}$ conformation, a perturbation was performed. Sodium was linearly perturbed to potassium during a thermodynamic integration run of $250 \mathrm{ps}$. The starting conformation was the most frequently sampled conformation of the 18-crown-6-sodium simulation (see the next subsection). After various dihedral transitions, the complex adopted, after only $57 \mathrm{ps,}$ the $\mathrm{D}_{3 d}$ conformation in which it stayed the rest of the time. So, even when the intermediate state in the perturbation (where only the radius of the com-

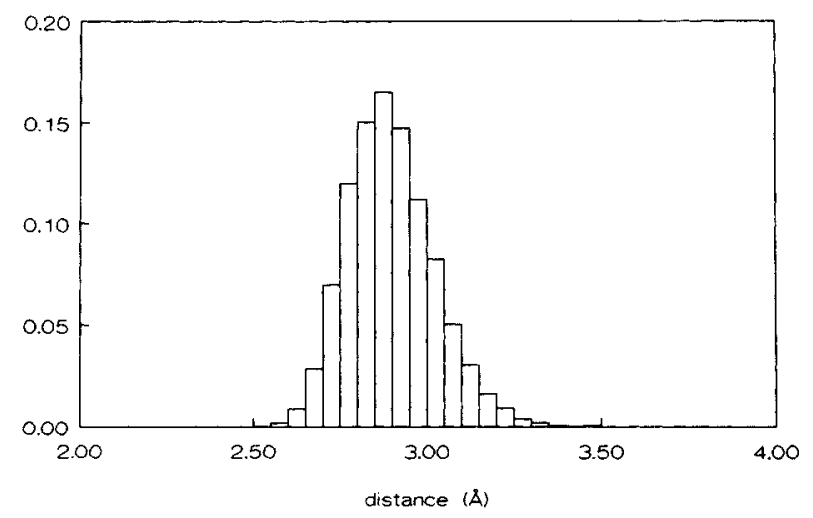

Figure 1. Normalized distribution of the oxygen-potassium distances for the 18 -crown- $6: \mathrm{K}^{+}$complex. Molecular dynamics simulation.

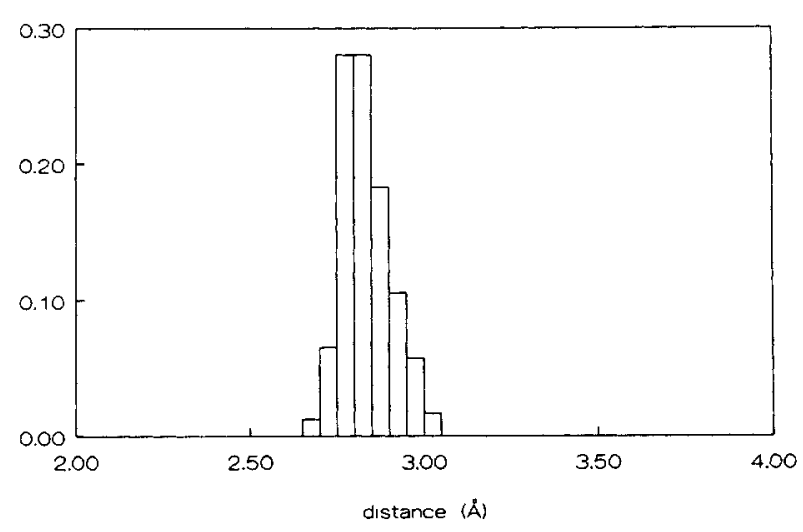

Figure 2. Normalized distribution of the oxygen-potassium distances for the 18-crown- $6: \mathrm{K}^{+}$complexes from the Cambridge Structural Database.

plexed ion was changed) still has more than $75 \%$ sodium character the macrocycle already adopts the $\mathrm{D}_{3 d}$ conformation. Similar conformational changes were observed during the same kind of perturbation calculations on the complex in nonaqueous solvents. ${ }^{12,13}$ It seems that the conformation of the crown ether is very sensitive to the radius of the ion.

A search in the CSD yielded 41 structures of 18crown- $6: \mathrm{K}^{+}$complexes, which all but one ${ }^{14}$ (code: $++00-00-00+00-00+0)$ had $D_{3 d}$ symmetry. Recently, the structure of yet another non- $\mathrm{D}_{3 d}$ potassium complex has been published ${ }^{15}$ (code: ++0 $0-00+0++00-00+0$ ). Although not sampled for the potassium complex, these conformations were sampled with high frequency for respectively the sodium complex and the urea complexes (see the second, fourth, and fifth subsections in this section and Tables II, V, and VII). Apparently, though, the $\mathrm{D}_{3 d}$ conformation is by far more favorable than any other conformation both in crystals and in the simulations.

Oxygen-potassium distances were calculated for both the simulation and the CSD data (see Figs. 1 and 2 and Table I). There is a slight discrepancy between the average for the simulation (2.91 $\mathrm{SD}=0.13 \AA$ ) and the average for the Database $(2.84 \AA$; SD $=0.07 \AA$ ). This may be ascribed to the parameter set used for the complex in the molecular dynamics run and to the neglect of crystal field effects. The internal pressure in the crystals will be responsible for some shortening of the distances.

The fact that no dihedral transitions were observed in the simulation may at first seem rather

Table I. Averaged oxygen-cation distances (in $\AA$ ) for the simulation and CSD data.

\begin{tabular}{ccc}
\hline Metal-Ion & MD Run & CSD \\
\hline $\mathrm{K}^{+}$ & $2.91(0.13)$ & $2.84(0.07)$ \\
$\mathrm{Na}^{+}$ & $2.56(0.20)$ & $2.66(0.14)$ \\
$\mathrm{Rb}^{+}$ & $2.99(0.11)$ & $2.96(0.06)$ \\
\hline
\end{tabular}

Standard deviations are given in brackets. 
Table II. Crown ether conformations with frequency $>100$ from the 18 -crown- $6: \mathrm{Na}^{+}$simulation.

\begin{tabular}{|c|c|c|c|c|c|c|c|c|}
\hline \multirow[b]{2}{*}{1} & \multicolumn{6}{|c|}{ Conformation } & \multirow{2}{*}{$\begin{array}{c}\text { Frequency } \\
10,957\end{array}$} & \multirow{2}{*}{$\frac{\% \text { Occurrence }}{36.5}$} \\
\hline & $t+0$ & $0++$ & $0+0$ & ++0 & $0-0$ & $0+0$ & & \\
\hline 2 & ++0 & $0-0$ & $0+0$ & ++0 & $0-0$ & $0+0$ & 5546 & 18.5 \\
\hline 3 & ++0 & $0-0$ & $0+0$ & $0--$ & $0-0$ & $0+0$ & 2262 & 7.5 \\
\hline 4 & $t+t$ & $0+0$ & $0--$ & $0-0$ & $0+0$ & $0--$ & 1227 & 4.1 \\
\hline 5 & $+t+$ & $0+0$ & $0--$ & $0-0$ & --0 & $0+0$ & 1108 & 3.7 \\
\hline 6 & $t+0$ & $0-0$ & $0+0$ & $0-0$ & $0++$ & $0+0$ & 1064 & 3.5 \\
\hline 7 & $0+0$ & $0-0$ & $0+0$ & $0-0$ & $0+0$ & $0-0$ & 1005 & 3.4 \\
\hline 8 & $t+0$ & $0-0$ & $0+0$ & $0--$ & $0++$ & $0+0$ & 965 & 3.2 \\
\hline 9 & $+t+$ & $0+0$ & $0-0$ & $0+0$ & $0-0$ & $0+0$ & 680 & 2.3 \\
\hline 10 & $t+t$ & $0+0$ & ++0 & $0-0$ & $0+0$ & $0--$ & 651 & 2.1 \\
\hline 11 & $++t$ & $0++$ & $0+0$ & $0-0$ & $0++$ & $0+0$ & 604 & 2.0 \\
\hline 12 & $+t 0$ & $0-+$ & $0+0$ & $0-0$ & $0++$ & $0+0$ & 328 & 1.1 \\
\hline 13 & $t+0$ & $0-0$ & $0+0$ & $0--$ & $0-+$ & $0+0$ & 321 & 1.0 \\
\hline 14 & $+t+$ & $0+0$ & ++0 & $0+0$ & $0-0$ & $0+0$ & 312 & 1.0 \\
\hline 15 & ++0 & $0-0$ & $0+0$ & ++0 & $0-0$ & --0 & 281 & 0.9 \\
\hline 16 & $+t+$ & $0+0$ & ++0 & $0-0$ & --0 & $0+0$ & 217 & 0.7 \\
\hline 17 & +++ & $0++$ & $0+0$ & $0-0$ & --0 & $0+0$ & 216 & 0.7 \\
\hline 18 & $t+0$ & $0++$ & $0+0$ & $0-0$ & -0 & $0+0$ & 210 & 0.7 \\
\hline 19 & $t+0$ & $0++$ & $0+0$ & ++0 & $0++$ & $0+0$ & 157 & 0.5 \\
\hline 20 & $t+0$ & $0-0$ & $0+0$ & +-0 & --0 & $0+0$ & 106 & 0.4 \\
\hline 21 & ++0 & $0+0$ & $0-0$ & $0+0$ & $0-0$ & $0+0$ & 105 & 0.4 \\
\hline
\end{tabular}

strange because Ratcliffe et al. ${ }^{16}$ found, from nuclear magnetic resonance (NMR) measurements in the solid state, a reorientational process reminding of the motion of a merry-go-round. The orientational adjustments were estimated to occur once every $10^{-5}-10^{-6} \mathrm{~s}$, much too slow to be seen on the ps time scale of the MD simulations. Mazor et al., ${ }^{13}$ who monitored the values of the dihedral angles for the potassium complex in methanol, did not find dihedral transitions too.

\section{8-Crown-6: $\mathrm{Na}^{+}$Complex}

For the sodium complex, an MD run with a total length of $15 \mathrm{~ns}$ was performed. In contrast to the potassium run (the previous subsection), numerous dihedral transitions were observed during this simulation. Of the 922 conformations that were generated, 158 were symmetry independent. The conformations with a frequency higher than 100 are listed in Table II. The CSD yielded 15 structures for the 18crown-6: sodium complex. These are listed in Table III. The first thing that can be noted is that the conformation with the highest frequency in the simu- lation is not found in the CSD, although structure 10 in Table III differs only in three dihedral angles from structure 1 in the simulation. Further, not all conformations from the Database were sampled in the MD run (numbers 3, 4, and 6 in Table III).

Oxygen-sodium distances are plotted in Figures 3 and 4 . Because the sodium cation is too small to fill the cavity of an 18-crown-6 molecule having a $\mathrm{D}_{3 d}$ conformation, the ring adopts a highly irregular conformation, in which some of the oxygen-cation distances become smaller whereas others tend to get larger. The average oxygen-metal distance is found to be smaller for the sodium complex than it is for the potassium complex. The distance plot gets distorted to the right. The latter is confirmed by the calculated skewness (see Table IV). The spreading in the distances is significantly larger when compared to the potassium complex (see Table I). The same features were observed by Mazor et al., ${ }^{13}$ who performed an extensive structural and thermodynamic analysis of the cationic selectivity of 18 crown-6 in methanol.

To substantiate the above findings, the conformations with $\mathrm{D}_{3 d}$ symmetry (1005 samples; see Table

Table III. Crown ether conformations of sodium complexes from CSD.

\begin{tabular}{|c|c|c|c|c|c|c|c|c|}
\hline & \multicolumn{6}{|c|}{ Conformation } & Frequency & No. in Simulation \\
\hline 1 & $0+0$ & $0-0$ & $0+0$ & $0-0$ & $0+0$ & $0-0$ & 6 & 7 \\
\hline 2 & $+t+$ & $0+0$ & ++0 & $0-0$ & --0 & $0+0$ & 1 & 16 \\
\hline 3 & $+t+$ & $0+0$ & $0--$ & ++0 & --0 & $0+0$ & 1 & - \\
\hline 4 & ++0 & ++0 & $0+0$ & $0-+$ & $+0-$ & -00 & 1 & - \\
\hline 5 & +++ & $0+0$ & ++0 & $0-0$ & $0-0$ & $0+0$ & 1 & 35 \\
\hline 6 & +++ & $0+0$ & ++0 & $0+0$ & $0+0$ & $0 \ldots$ & 1 & - \\
\hline 7 & ++0 & $0-0$ & $0+0$ & $0--$ & $0-0$ & $0+0$ & 1 & 3 \\
\hline 8 & ++0 & $0+0$ & $0-0$ & $0+0$ & $0-0$ & $0+0$ & 1 & 21 \\
\hline 9 & $+t+$ & $0+0$ & ++0 & $0-0$ & $0+0$ & $0--$ & 1 & 10 \\
\hline 10 & ++0 & $0-0$ & $0+0$ & $0+0$ & $0-0$ & $0+0$ & 1 & 47 \\
\hline
\end{tabular}




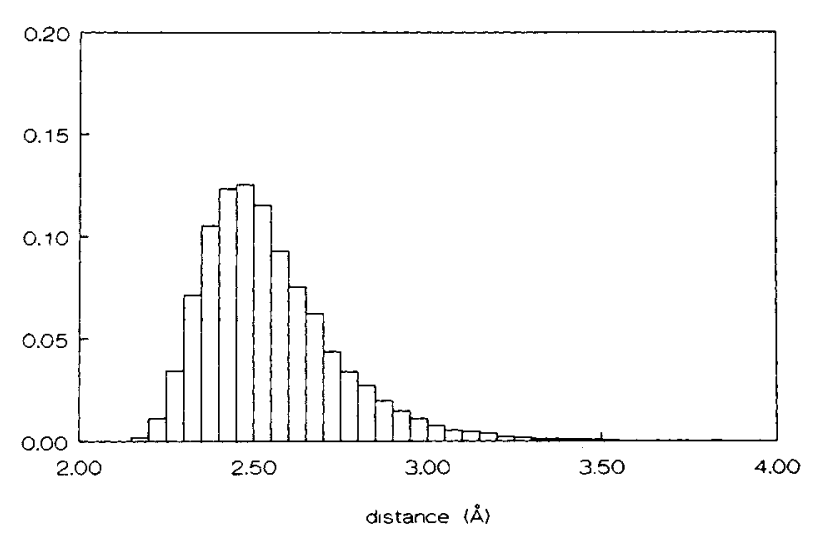

Figure 3. Normalized distribution of the oxygen-sodium distances for the 18-crown-6: $\mathrm{Na}^{+}$complex. Molecular $\mathrm{dy}$ namics simulation.

II) were separated from the other conformations and compared to those of the potassium complex. In Figure 5, the distribution of the $\mathrm{O}-\mathrm{Na}^{+}$distances for this selection is given. The average is $2.81 \AA$ ( $\mathrm{SD}=0.29 \AA$ ). The spreading in this distribution is much larger than in the case of potassium (see Fig. 1). Compared to Figure 3, the maximum is clearly shifted toward higher values. Although some oxygen-sodium distances can get fairly short for the $D_{3 d}$ conformation, it seems beneficial for the crown to adopt an irregular conformation in which the electrostatic interaction between oxygen and sodium is more favorable.

\section{8-Crown-6: $\mathbf{R b}^{+}$Complex}

In this simulation with a length of $7 \mathrm{~ns}$, again no dihedral transitions were observed. As in the case of the potassium complex, the crown ether stayed in the $\mathrm{D}_{3 d}$ conformation all the time. In view of the results of the perturbation in the first subsection in this section, this might have been expected because the radius of the $\mathrm{Rb}^{+}$-ion is slightly larger than that of the potassium ion.

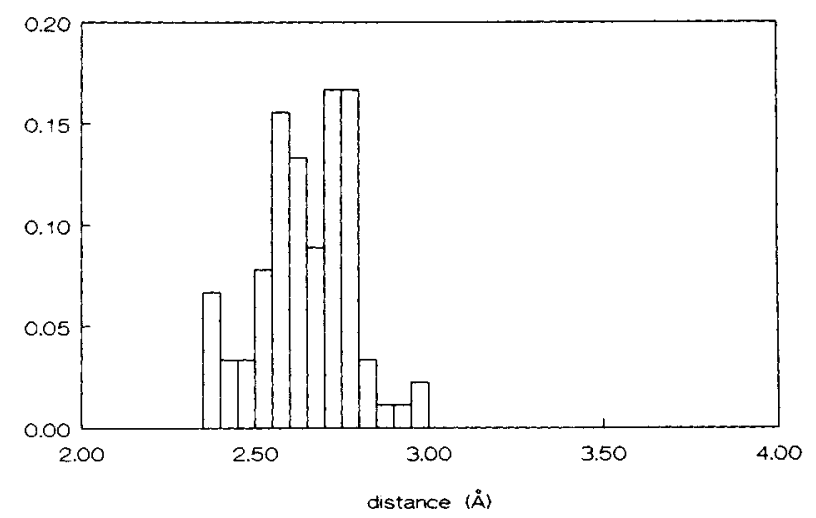

Figure 4. Normalized distribution of the oxygen-sodium distances for the 18-crown-6: $\mathrm{Na}^{+}$complexes from the Cambridge Structural Database.
Table IV. Calculated skewness of the cation-oxygen distance plots.

\begin{tabular}{lcr}
\hline \multicolumn{1}{c}{ Metal-Ion } & MD & CSD \\
\hline $\mathrm{K}^{+}$ & 0.28 & 0.06 \\
$\mathrm{Na}^{+}$ & 1.17 & -0.27 \\
$\mathrm{Na}^{+}$(only $\left.\mathrm{D}_{3 d}\right)$ & 0.56 & - \\
$\mathrm{Rb}^{+}$ & 0.23 & - \\
\hline
\end{tabular}

The CSD yielded three structures, all three with $\mathrm{D}_{3 d}$ symmetry. The average oxygen-cation distances match well (see Table I and Fig. 6); however the number of entries in the CSD is too low to allow for reliable statistics. For the largest ion, $\mathrm{Rb}^{+}$, the skewness (Table IV) is the smallest.

\section{8-Crown-6: Urea $_{2}$ Complex}

First, the organic complex of 18-crown-6 with two urea molecules was simulated. One urea was positioned above the ring and the other below the ring (see Fig. 7). This complex was simulated for 6 ns. During these $6 \mathrm{~ns}, 292$ conformations were sampled, 67 of which were symmetry independent. The conformations with a frequency larger than 50 are listed in Table $\mathrm{V}$.

The $\mathrm{D}_{3 d}$ conformation is by far the most frequently sampled conformation (51.3\%). This was also the most frequently sampled conformation of the uncomplexed crown ether in aqueous solution, ${ }^{3}$ where solvent molecules form hydrogen bridges with both sides of the macrocycle. Dang and Kollman, ${ }^{17}$ however, who calculated the potential of mean force for the association of $\mathrm{K}^{+}$with 18-crown- 6 in water, found that the symmetry of the crown during the association process was mainly of $C_{1}$ and $C_{2}$ symmetry.

In the CSD, there are a number of complexes with organic molecules (some of which are charged) that all exhibit one of two conformations. The most frequently occurring conformation has $\mathrm{D}_{3 d}$ symmetry

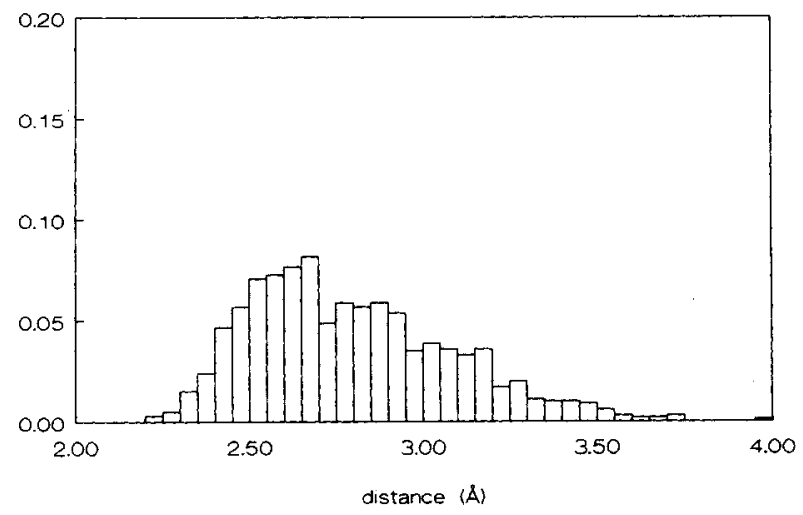

Figure 5. Normalized distribution of the oxygen-sodium distances for the samples from the simulation with $D_{3 d}$ symmetry. 


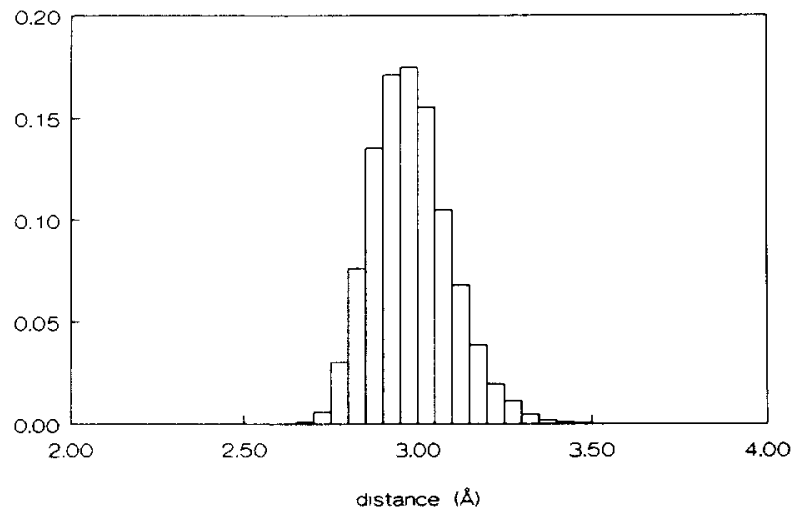

Figure 6. Normalized distribution of the oxygen-rubidium distances for the 18 -crown-6: $\mathrm{Rb}^{+}$complex. Molecular dynamics simulation.

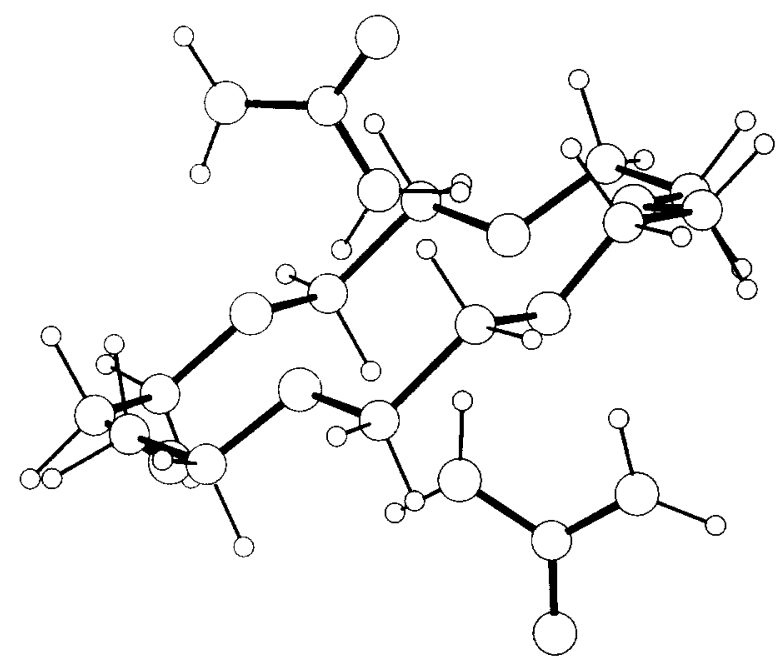

Figure 7. Snapshot of the 18-crown-6: urea 2 complex.

and the other is a conformation that only appears with organic molecules (code: $++00-00-0--0$ $0+00+0$ ). This one is not sampled in the simulation. The second most sampled conformation, though $(20.4 \%)$, differs only in two dihedral angles from the "organic conformation" in the CSD.
It was observed that hydrogen bonds between the urea and the crown ether were broken and then formed again with other oxygens of the macrocycle. Also, three-center hydrogen bonds were observed. This breaking and reforming of hydrogen bonds is reflected by the fact that for each of the nitrogen atoms of one of the urea molecules the distances to the different oxygens of the ring are on average approximately the same (see Table VI). It can also be seen from this table that both nitrogens behave equivalently in this respect. The unfavorable dipole interactions between the urea molecules make it highly probable that the urea molecules are bonded to different sites of the crown ether, which may be why a significant part of the ring behaves rather rigidly. This is expressed by the fact that only 67 unique conformations were sampled in $6 \mathrm{~ns}$, which should be compared with the 852 symmetry-independent conformations of the 1390 that were sampled during $6 \mathrm{~ns}$ in the case of the free crown. (The simulation of the uncomplexed 18-crown-6, with a total length of $15 \mathrm{~ns}$, is used here only as a reference to compare its flexibility with that of the urea complexes.)

One might surmise that the breaking and forming of hydrogen bonds is much too slow a process to be seen on the time scale of the simulations. In these urea complexes (see also the next subsection), however, more than one oxygen might play a role in the hydrogen bonding process by which the barrier between two hydrogen-bonded conformations is considerably lowered.

In Figure 8, the $\mathrm{O}-\mathrm{N}$ distances are plotted for one of the oxygens from the ring and one of the nitrogens of one of the urea molecules. The first thing that can be noticed is that it has two maxima, one at $3.3 \AA$ and the other at $5.1 \AA$. The first maximum is from direct hydrogen bonding. The second is due to a specific position of the urea molecule with respect to the ring when the other nitrogen is involved in hydrogen bonding. To test if this second maximum is in a way conformational dependent, the $\mathrm{D}_{3 d}$ con-

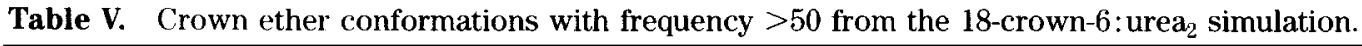

\begin{tabular}{|c|c|c|c|c|c|c|c|c|}
\hline \multirow[b]{2}{*}{1} & \multicolumn{6}{|c|}{ Conformation } & \multirow{2}{*}{$\frac{\text { Frequency }}{6151}$} & \multirow{2}{*}{$\frac{\% \text { Occurrence }}{51.3}$} \\
\hline & $0+0$ & $0-0$ & $0+0$ & $0-0$ & $0+0$ & $0-0$ & & \\
\hline 2 & ++0 & $0-0$ & $0+0$ & $0-0$ & $0+0$ & $0+0$ & 2453 & 20.4 \\
\hline 3 & $t+0$ & $0-0$ & $0-0$ & $0+0$ & $0-0$ & $0+0$ & 939 & 7.8 \\
\hline 4 & $0+0$ & $0+0$ & $0-0$ & $0+0$ & $0+0$ & $0-0$ & 481 & 4.0 \\
\hline 5 & $0+0$ & $0+0$ & $0-0$ & $0+0$ & $0-0$ & $0-0$ & 427 & 3.6 \\
\hline 6 & ++0 & $0-0$ & $0+0$ & $0+0$ & $0-0$ & $0+0$ & 288 & 2.4 \\
\hline 7 & ++0 & $0-0$ & $0+0$ & $0-0$ & $0-0$ & -+0 & 137 & 1.1 \\
\hline 8 & ++0 & $0-0$ & $0-0$ & $0++$ & $0+0$ & $0+0$ & 136 & 1.1 \\
\hline 9 & ++0 & $0-0$ & $0+0$ & $0-0$ & --0 & $0+0$ & 127 & 1.1 \\
\hline 10 & ++0 & $0-0$ & $0++$ & $0+0$ & $0-0$ & $0+0$ & 96 & 0.8 \\
\hline 11 & ++0 & $0-0$ & $0+0$ & $0-0$ & $0-0$ & $0+0$ & 78 & 0.7 \\
\hline 12 & ++0 & $0-+$ & $0+0$ & $0+0$ & $0-0$ & $0+0$ & 71 & 0.6 \\
\hline 13 & +-0 & $0+0$ & $0-0$ & $0+0$ & $0-0$ & $\begin{array}{lll}0 & 0 & 0\end{array}$ & 63 & 0.5 \\
\hline 14 & ++0 & $0-0$ & $0++$ & $0+0$ & $0+0$ & $0+0$ & 56 & 0.5 \\
\hline 15 & ++0 & $0-0$ & $0+0$ & ++0 & $0-0$ & $0+0$ & 51 & 0.4 \\
\hline
\end{tabular}


Table VI. Calculated average values for all $12 \mathrm{O}-\mathrm{N}$ distances $(\AA)$ between 18 -crown- 6 and one of the urea molecules.

\begin{tabular}{cccccc}
\hline $\mathrm{O} 1-\mathrm{N} 1$ & $\mathrm{O} 2-\mathrm{N} 1$ & $\mathrm{O} 3-\mathrm{N} 1$ & $\mathrm{O} 4-\mathrm{N} 1$ & $\mathrm{O} 5-\mathrm{N} 1$ & $\mathrm{O} 6-\mathrm{N} 1$ \\
\hline 4.0982 & 4.1808 & 4.0714 & 4.1218 & 4.1776 & 4.1316 \\
\hline $\mathrm{O} 1-\mathrm{N} 2$ & $\mathrm{O} 2-\mathrm{N} 2$ & $\mathrm{O} 3-\mathrm{N} 2$ & $\mathrm{O} 4-\mathrm{N} 2$ & $\mathrm{O} 5-\mathrm{N} 2$ & $\mathrm{O} 6-\mathrm{N} 2$ \\
\hline 4.0437 & 4.1101 & 4.0089 & 4.0784 & 4.1363 & 4.0840 \\
\hline
\end{tabular}

formations were separated from the rest. The $\mathrm{O}-\mathrm{N}$ distances for the two sets of conformations are plotted in Figures 9 and 10. There seems to be no connection between the second maximum in Figure 8 and the type of conformation.

\section{8-Crown-6: Urea Complex}

The complex of 18-crown- 6 with one urea was simulated for $13 \mathrm{~ns}$. Of the 1150 conformations that were generated, 531 were unique. The conformations with a frequency larger than 125 are listed in Table VII. Again, the $\mathrm{D}_{3 d}$ conformation has the highest frequency $(42.7 \%)$. The second most sampled conformation is also the same as for the 18-crown-6: urea $_{2}$ complex and differs in two dihedral angles from the "organic structure" in the Database (see the previous section).

The 18-crown-6: urea complex is the only complex that adopted the $C_{i}$ conformation (code: $+-00+0$ $000-+00-0000$; freq. $=0.2 \%$ ). In the case of the free crown, the $C_{i}$ conformation is the most frequently sampled conformation (11.5\%). This elliptical conformation, in which the size of the cavity is reduced in favor of better intramolecular interactions, is also the one the free crown displays in the solid state. (This is in accordance with earlier studies on this subject. ${ }^{4,8}$ ) The fact that the $C_{i}$ conformation, which is normally not observed for complexes of 18 crown-6, does occur for the urea complex may be

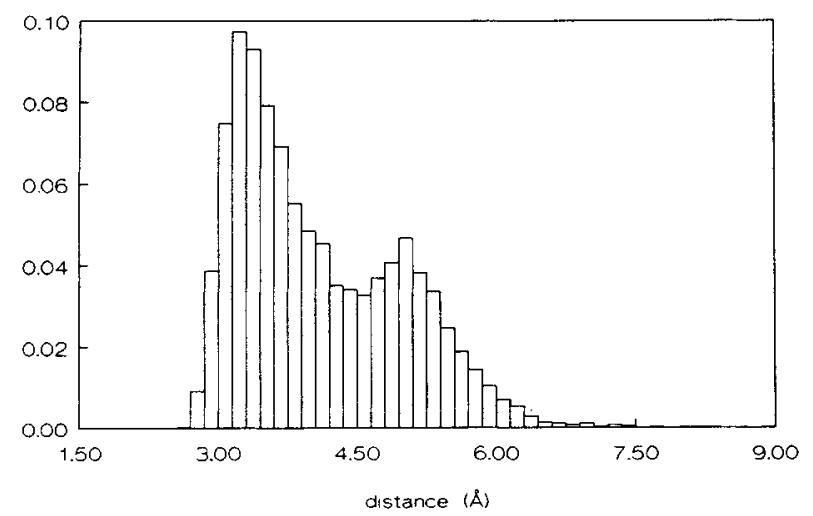

Figure 8. Normalized distribution of the oxygen-nitrogen distances for the simulated 18-crown-6: urea $_{2}$ complex.

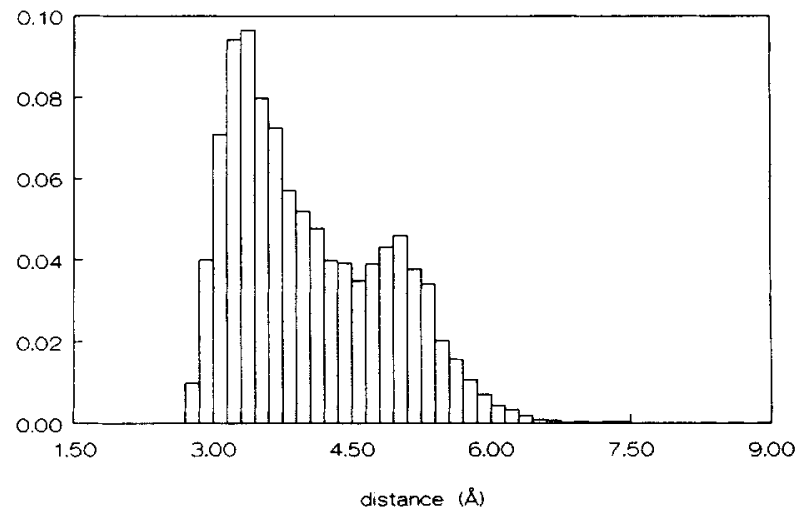

Figure 9. Normalized distribution of the oxygen-nitrogen distances for the 18-crown-6: urea 2 complex. Only samples with $\mathrm{D}_{3, d}$ symmetry.

caused by the absence of a more complex hydrogen bonding network that would have been the case when more molecules would have been involved. In the case of the metal ions, it is obvious that this $C_{i}$ conformation is not possible.

The number of different conformations sampled in this simulation is an indication that in this complex the crown is much less rigid than in the case with the two urea but it is by far not as flexible as the uncomplexed crown. For the free crown ether after $13 \mathrm{~ns}, 3089$ conformations were generated, 1634 of which were symmetry independent.

When the oxygen-nitrogen distances are plotted, again two maxima are observed (Fig. 11) at the same distances as for the 18-crown-6: urea 2 complex. Separating the $\mathrm{D}_{3 d}$ conformations from the rest of the conformations gives the same result as in the previous case. The type of conformation is not responsible for the appearance of the second maximum. Neither does there seem to exist any kind of correlation between both urea molecules in the 18 . crown-6: urea $_{2}$ complex that causes the appearance of the second peak.

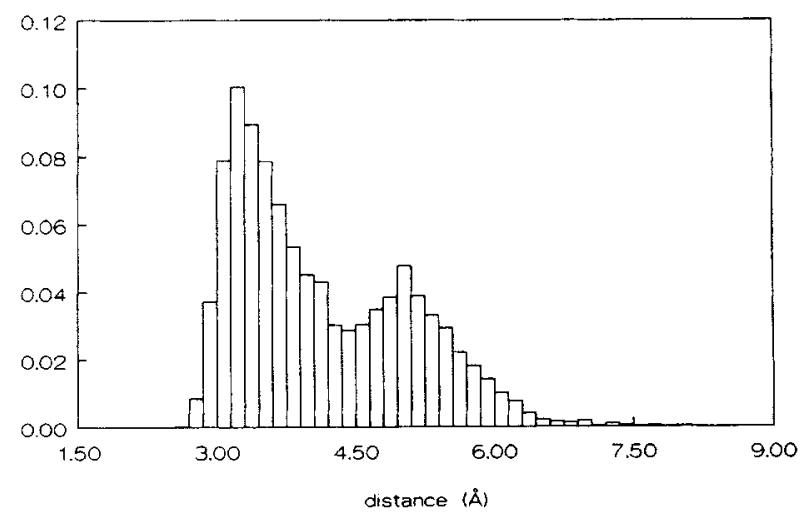

Figure 10. Normalized distribution of the oxygen-nitrogen distances for the 18-crown-6: urea ${ }_{2}$ complex. Samples with symmetry other than $\mathrm{D}_{3 d}$ symmetry. 
Table VII. Crown ether conformations with frequency $>125$ from the 18-crown-6: urea simulation.

\begin{tabular}{|c|c|c|c|c|c|c|c|c|}
\hline & & & Conf & nation & & & Frequency & $\%$ Occurrence \\
\hline 1 & $0+0$ & $0-0$ & $0+0$ & $0-0$ & $0+0$ & $0-0$ & 11,089 & 42.7 \\
\hline 2 & ++0 & $0-0$ & $0+0$ & $0-0$ & $0+0$ & $0+0$ & 3934 & 15.1 \\
\hline 3 & +-0 & $0+0$ & $0-0$ & $0+0$ & $0-0$ & 000 & 1289 & 5.0 \\
\hline 4 & ++0 & $0-0$ & $0-0$ & $0+0$ & $0-0$ & $0+0$ & 802 & 3.1 \\
\hline 5 & ++0 & $0-0$ & $0+0$ & $0-0$ & $0+-$ & $0-0$ & 544 & 2.1 \\
\hline 6 & ++0 & $0-0$ & $0+0$ & $0--$ & $0-0$ & $0+0$ & 540 & 2.1 \\
\hline 7 & +-0 & $0+0$ & $0+0$ & +-0 & $0+0$ & $0+0$ & 408 & 1.6 \\
\hline 8 & ++0 & 000 & -+0 & $0-0$ & $0+0$ & $0+0$ & 384 & 1.5 \\
\hline 9 & +-0 & $0+0$ & $0-0$ & $0-0$ & $0+0$ & $0+0$ & 332 & 1.3 \\
\hline 10 & ++0 & $0-0$ & $0+0$ & ++0 & $0-0$ & $0+0$ & 277 & 1.1 \\
\hline 11 & $0+0$ & $0+0$ & $0-0$ & $0+0$ & $0+0$ & $0-0$ & 252 & 1.0 \\
\hline 12 & $0+0$ & $0+0$ & $0-0$ & $0+0$ & $0-0$ & $0-0$ & 221 & 0.9 \\
\hline 13 & +-0 & $0+0$ & $0+0$ & $0-0$ & $0+0$ & $0+0$ & 213 & 0.8 \\
\hline 14 & ++0 & $0-+$ & $0+0$ & $0+0$ & $0-0$ & $0+0$ & 174 & 0.7 \\
\hline 15 & ++0 & $0-0$ & $0+0$ & $0--$ & $\begin{array}{lll}0 & 0 & 0\end{array}$ & -+0 & 171 & 0.7 \\
\hline 16 & ++0 & $0-0$ & $0-0$ & $0+-$ & 000 & $0+0$ & 169 & 0.7 \\
\hline 17 & ++0 & $0-0$ & $0+0$ & $0+0$ & $0-0$ & $0+0$ & 167 & 0.6 \\
\hline 18 & ++0 & $0+0$ & +-0 & $0+0$ & $0-0$ & $0+0$ & 158 & 0.6 \\
\hline 19 & ++0 & $0-0$ & $0+0$ & $0-0$ & $0-0$ & -+0 & 140 & 0.5 \\
\hline 20 & $t+0$ & $0-0$ & $\begin{array}{lll}0 & 0 & 0\end{array}$ & +-0 & $0+0$ & $0+0$ & 127 & 0.5 \\
\hline
\end{tabular}

\section{DISCUSSION AND CONCLUSIONS}

The aim of this study was to investigate whether there exists a similarity between the occurrences of conformations of 18-crown- 6 complexes in their crystal phases and in MD simulations of the isolated complexes.

It turned out that during the simulation of the uncomplexed crown ether many conformations were generated, which means that the free crown is a highly flexible molecule. The most frequently sampled conformation was the $C_{i}$ conformation, which is the one the free crown adopts in the solid state.

For each of the 18-crown-6 complexes with $\mathrm{K}^{+}$ and $\mathrm{Rb}^{+}$, only one conformation was sampled, the one that is also found in the CSD. In the case of sodium, the situation is completely different. Both the MD study and CSD analysis show that many conformations exist within a narrow energy range. A marked difference is the fact that the most fre-

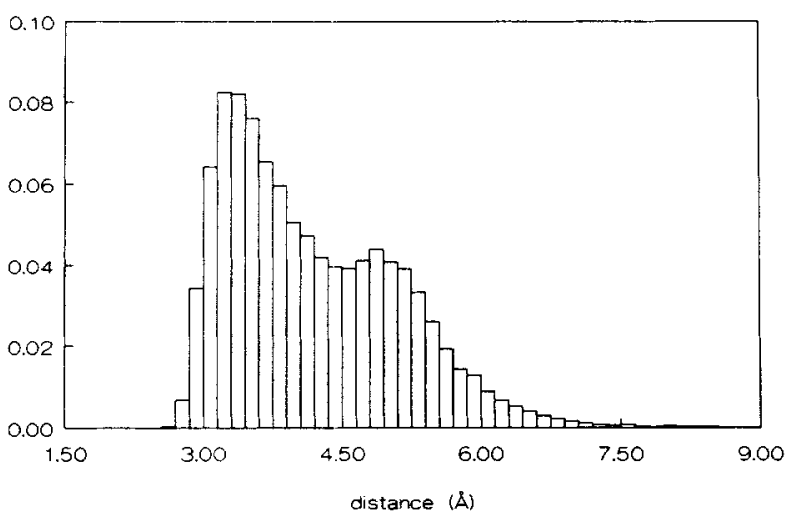

Figure 11. Normalized distribution of the oxygen-nitrogen distances for the simulated 18-crown-6: urea complex. quently sampled conformation during the MD run was not found in the Database (see below).

The above results are intuitively clear. The Coulombic forces tend to make the metal-oxygen distances as small as possible with the constraint that the internal energy of the ring should not become appreciably larger than in its lowest-energy state. In the case of the bigger ions, the crown can achieve this easily by choosing the $\mathrm{D}_{3 d}$ conformation that has the lowest possible strain energy. In the case of the smaller sodium ion, the molecule uses its flexibility to lower the total energy as much as possible by choosing a sequence of low-symmetry conformations. One could say that the two big ions are more complementary to the crown than the sodium ion.

The results of the two urea simulations are similar. The two most frequently sampled conformations, which account for more than half of all samples, are the same in both cases. Further, the features of the nitrogen-oxygen distance distributions are the same for both simulations. These results are in good agreement with similar results obtained from the CSD. The main discrepancy is that the organic conformation in the CSD does not occur in the MD simulation but differs in two dihedral angles from the second most sampled conformation.

From the number of conformations that were sampled in the simulations, it is seen that the macrocycle in both of the urea complexes is much more rigid than the free crown. Apparently, the hydrogen bonding between urea and the crown prevents part of the ring from taking more than one conformation. Adding a second urea molecule stiffens the remaining part of the crown such that only a few conformational transitions remain, and the macrocycle adopts a more or less flat conformation. 
A comparable situation as with the urea complexes occurs in crystals. The complex is surrounded by many other molecules that together tend to stabilize a flat conformation. In the case of sodium, this effect opposes the wrapping of the crown ether around the ion and makes the most frequently sampled MD conformation less probable in a crystal environment. Moreover, this also explains why most crystal structures adopt the $\mathrm{D}_{3 d}$ conformation (see Table III). This is acknowledged by a previous search in the CSD by Fyles and Gandour, ${ }^{18}$ who paid attention to the crystal packing arrangements. From their article, it can be seen that in crystals of both potassium and sodium complexes other molecules or fragments, i.e, solvent molecules and counterions, are often involved in the coordination. This results for most of the complexes in coordination numbers in the range $7-9$. In this respect, there is not much difference between the two cations (see also ref. 19).

\section{References}

1. C.J. Pedersen, J. Am. Chem. Soc., 89, 2495 (1967).

2. J. van Eerden, S. Harkema, and D. Feil, J. Phys. Chem., 92, 5076 (1988).

3. T.P. Straatsma and J.A. McCammon, J. Chem. Phys., 91, 3631 (1989).
4. Y. Sun and P.A. Kollman, J. Comp. Chem., 13, 33 (1992).

5. F.H. Allen, O. Kennard, and R. Taylor, Acc. Chem. Res., 16, 146 (1983).

6. W.F. van Gunsteren and H.J.C. Berendsen, Groningen Molecular Simulation Library, Groningen, The Netherlands, 1987.

7. S.J. Weiner, P.A. Kollman, D.T. Nguyen, and D.A. Case, J. Comp. Chem., 7, 230 (1986).

8. G. Wipff, P. Weiner, and P.A. Kollman, J. Am. Chem. Soc., 104, 3249 (1982).

9. A.T. Hagler, E. Huler, and S. Lifson, J. Am. Chem. Soc., 96, 5319 (1976).

10. E.S. Boek, W.J. Briels, J, van Eerden, and D. Feil, J. Chem. Phys., 96, 7010 (1992).

11. J. Ryckaert, G. Ciccoti, and H.J.C. Berendsen, J. Comp. Phys., 23, 327 (1977).

12. M.H. Mazor, J.A. McCammon, and T.P. Lybrand, J. Am. Chem. Soc., 111, 55 (1989).

13. M.H. Mazor, J.A. McCammon, and T.P. Lyrand, J. Am. Chem. Soc., 112, 4411 (1990).

14. M.G.B. Drew, K.C. Lee, and K.F. Mok, Inorg. Chim. Acta, 115, 39 (1989).

15. K.M. Doxsee, H.R. Wierman, and T.J.R. Weakley, J. Am. Chem. Soc., 114, 5165 (1992).

16. C.I. Ratcliffe, J.A. Ripmeester, G.W. Buchanan, and J.K. Denike, J. Am. Chem. Soc., 114, 3294 (1992).

17. L.X. Dang and P.A. Kollman, J. Am. Chem. Soc., 112, 5716 (1990).

18. T.M. Fyles and R.D. Gandour, J. Inclus. Phenom., 12, 313 (1992).

19. M. Dobler, Chimia, 38, 415 (1984). 